
\title{
$\begin{array}{ll}\text { Research Square } & \begin{array}{l}\text { Preprints are preliminary reports that have not undergone peer review. } \\ \text { They should not be considered conclusive, used to inform clinical practice, } \\ \text { or referenced by the media as validated information. }\end{array}\end{array}$
}

\section{Lipid Reprogramming Induced by TFEB-ERRa Axis Enhanced Membrane Fluidity to Promote EC Progression}

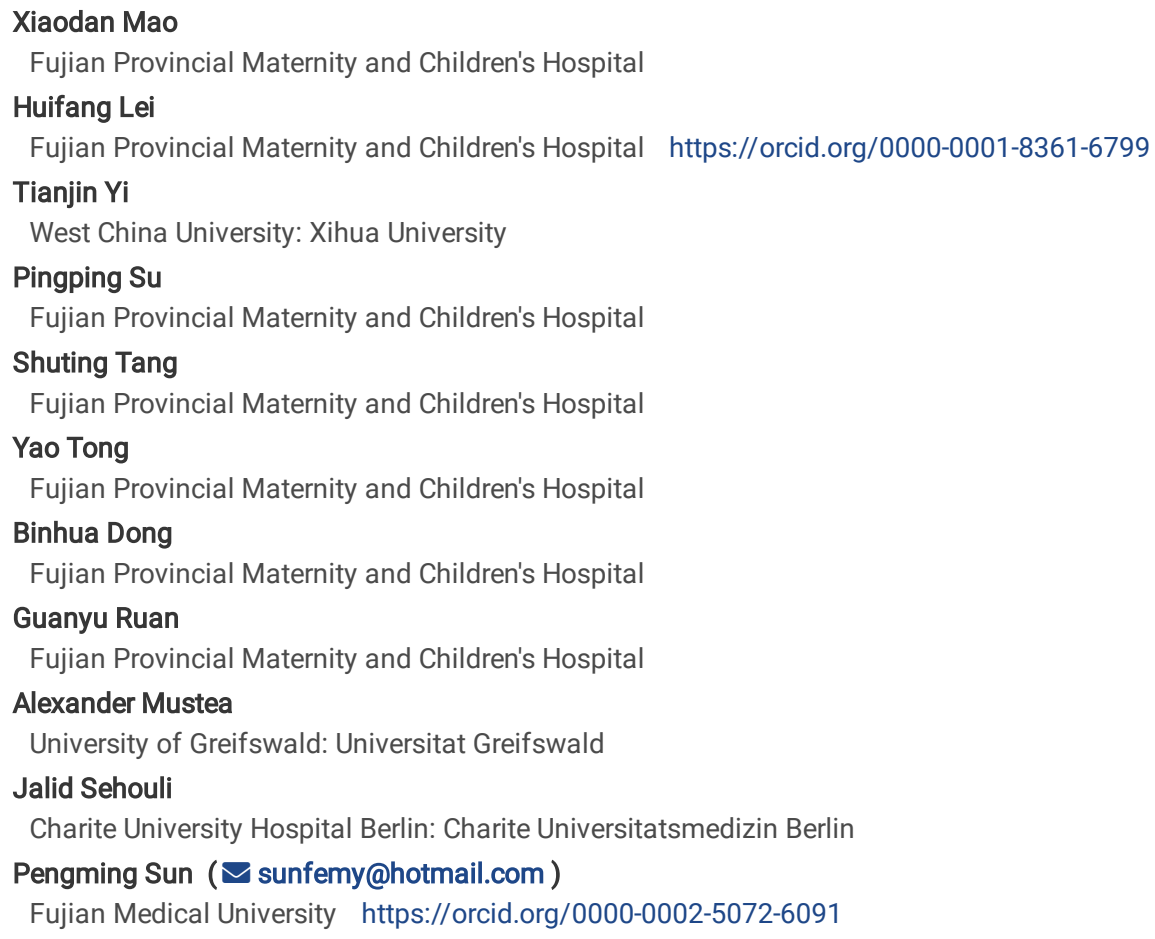




\section{Abstract}

Background: Endometrial cancer (EC) is one of the most common tumors in women. Estrogen-related receptor a (ERRa) has been reported to play a critical role in EC progression. However, the underlying mechanism of ERRa-mediated lipid reprogramming in EC remains elusive. Here, we show that transcription factor EB (TFEB)-ERRa axis promote lipid reprogramming to enhance invasion and metastasis of EC.

Methods: TFEB and ERRa were analyzed and validated by RNA-sequencing data of EC tissues from the Cancer Genome Atlas (TCGA). TFEB-ERRa axis were assessed by dual-luciferase reporter and chromatin immunoprecipitation quantitative polymerase chain reaction (ChIP-qPCR). The mechanism of TFEB-ERRa was investigated using loss-of-function and gain-of-function assays in vitro. Lipidomics and proteomics were performed to identify the ERRa-related lipid metabolism pathway. Furthermore, immunohistochemistry and lipidomics were performed in the tissues to verify the ERRa-related lipids.

Results: Both TFEB and ERRa were highly expression in EC patients and related to EC progression. ERRa was the directly target of TFEB to mediate EC lipid metabolism. Lipidomics assays demonstrated that ERRa mainly effects on glycerophospholipids (GPs) and phosphatidylcholine (PC) and significantly elevates the ratio of $\mathrm{PC} /$ sphingomyelin (SM) in EC cells, which indicated the enhanced membrane fluidity. Specifically, ERRa induced unsaturated fatty acid (UFA)-containing PCs, phosphatidylglycerol (PGs), SMs etc. Combined proteomics analysis revealed the increase of UFA-containing GPs mainly related to mitochondrial function. Then, the levels of maximum oxygen consumption rates (OCRs), adenosine triphosphate (ATP) and lipid metabolism-related genes acc, fasn, acadm were found to be positively correlated with TFEB/ERRa expression. Mechanistically, our functional assays indicate that TFEB promoted EC cell migration in a ERRa-dependent manner by epithelial-mesenchymal transformation (EMT) signaling. Consistent with in vitro, higher PC(18:1/18:2)+HCOO were found in EC patients and those who with higher expression of TFEB/ERRa had a deeper myometrial invasion and lower serum high-density lipoprotein (HDL). Moreover, $\mathrm{PC}(18: 1 / 18: 2)+\mathrm{HCOO}$ was an independent risk factor of the patients with lymph node metastasis and positively related to ERRa.

Conclusion: Lipid reprogramming induced by TFEB-ERRa axis increases UFA- containing PC, PG and SM, which enhanced membrane fluidity via EMT signaling to promote EC progression. Of note, $\mathrm{PC}(18: 1 / 18: 2)+\mathrm{HCOO}$ was the ERRa-associated potential predictor of EC metastasis.

\section{Background}

High body mass index (BMI) influences the current and future health of patients and is considered one of the top 5 global causes of death for females ${ }^{1}$. Among all female malignancies, endometrial cancer (EC) is most strongly associated with obesity, and obesity has been considered a very important risk factor for EC in postmenopausal women ${ }^{2}$. EC is the sixth most common cancer in women and the second most common gynecological malignancy globally. There were approximately 66,570 new cases and 12,940 reported deaths due to EC in the United States in $2021^{3}$. The incidence of EC has also markedly increased in China recently. The age-standardized incidence of EC was 63.4 per 100,000, the mortality rate was 21.8 per 100,000 , and the 5 -year relative survival was $72.8 \%$ from $2012-2015^{4}$. Considering the accompanying symptoms of overweight and diabetes in EC patients, tumor lipid metabolism has been the research focus. However, the mechanism of lipid metabolism in EC is still unclear.

Estrogen-related receptor a (ERRa, NR3B1, ESRRA) is a constitutively active ligand-independent orphan nuclear receptor that belongs to the nuclear receptor superfamily ${ }^{5}$. As a transcription factor, ERRa combines with its acknowledged coactivator peroxisome proliferator-activated receptor (PPAR) coactivator-1a (PGC-1a) and plays a central role in the regulation of cellular oxidative phosphorylation and liposome metabolism, resulting in many biological functions ${ }^{6,7}$. Consistent with an increasing number of studies on breast cancer ${ }^{8}$, colon cancer ${ }^{9}$ and ovarian cancer ${ }^{10}$, our previous works confirmed that high expression of ERRa was significantly related to a poor prognosis in $\mathrm{EC}^{10}$. Moreover, overexpression of ERRa can downregulate the expression of E-cadherin while upregulating the expression of vimentin and inducing epithelial-mesenchymal transformation (EMT), which promotes invasion and migration, indicating that it may be a new biomarker for predicting the risk of deep myometrial invasion and metastasis ${ }^{11}$. After targeted inhibition of ERRa by small interfering RNA (siRNA) or antagonist XCT790, transcription factor EB (TFEB) was identified as a potential interacting protein by a DNA/protein high-throughput assay ${ }^{12}$. TFEB, a master regulator of lysosomal biogenesis and autophagy, was found to have a crucial pathogenic role in different tumors ${ }^{13}$. Several recent studies have also focused on the function of TFEB in tumor cell metabolism. Carmine et al suggested that TFEB might be a novel therapeutic target for disorders of lipid metabolism, such as fatty liver disease, and that TFEB exerts global transcriptional control on lipid catabolism via PGC-1a and PPARa ${ }^{14}$. However, there were only very few reports about the association between TFEB and ERRa aside from those published by our team.

The EMT program is related to lipid remodeling of the cell membrane ${ }^{15}$. The fatty acyl moieties of membrane phospholipids exhibit considerable diversity in chain length and different degrees of saturation, which determine the biophysical properties of membranes, including their fluidity, curvature, and subdomain architecture $^{16}$. The major structural phospholipids in mammalian membranes are glycerophospholipids (GPs), among which phosphatidylcholine (PC) is the most abundant in mammalian cell membranes and subcellular organelles, accounting for $40-50 \%$ of total phospholipids ${ }^{17}$. The saturability of PC affects the plasma membrane of tumor cells to sustain oncogenic activity in a wide variety of cancers ${ }^{16,18}$. Lin et al showed that the length of the fatty acid chain in the membrane modulated plasma membrane fluidity and invasion of liver cancer ${ }^{19}$. The roles of lipid metabolism in the function of the membrane in EC still need to be unveiled. Interestingly, both ERRa and TFEB were reported to be involved in the lipid remodeling signaling pathway.

How ERRa and TFEB play roles in lipid metabolism and how this mechanism affects malignant cell metastasis and invasion still need more research. We hypothesized that TFEB-ERRa signaling, which regulates lipid metabolism, extensively affects membrane function to promote EC invasion and metastasis. In this work, our discovery discusses the crosstalk between TFEB and ERRa and their coregulation of FA metabolism to promote invasion and metastasis in EC.

\section{Materials And Methods}




\section{Cell lines and cell culture}

Human KLE endometrial adenocarcinoma cells were obtained from the Shanghai Cell Biological Research Institute (Shanghai, China), and ECC-1 cells were acquired from the American Type Culture Collection (ATCC, Manassas, VA, USA). KLE cells are ERa-, while ECC-1 cells are ERa + . KLE and ECC-1 cells were thawed and cultured in DMEM/F12 medium with $0.005 \mathrm{mg} / \mathrm{ml}$ insulin, $1 \%$ antibiotic-antimycotic solution, and $10 \%$ fetal bovine serum (FBS) at $37^{\circ} \mathrm{C}$ in $5 \%$ $\mathrm{CO}_{2}$. Cells treated with XCT790 (Sigma-Aldrich, St. Louis, MO, USA) were incubated in phenol red-free medium (Thermo Fisher) containing $1 \%$ serum replacement 2 (Sigma-Aldrich). ECC-1 and KLE ECs were incubated with $10 \mu \mathrm{M} \mathrm{XCT790} \mathrm{(in} \mathrm{dimethyl} \mathrm{sulfoxide} \mathrm{[DMSO];} \mathrm{Sigma-Aldrich)} \mathrm{or} \mathrm{DMSO} \mathrm{(control)} \mathrm{for}$ 24 hours. Lentiviral vectors expressing siRNAs targeting TFEB (named TFEB-KD) and ERRa (named ERRa-KD) were constructed. The following siRNA target sequence in the TFEB gene (GenBank accession No. NM_013261) was selected: 5'-GAG ACG AAG GTT CAA CAT CAA-3'. The siERRa sequence was 5'-GAG CGA GAG GAG TAT GTT CTA-3'. The lentiviral vector used to overexpress TFEB (named TFEB-OV, GenBank accession NM_001167827.3) and ERRa (named ERRa-OV, GenBank accession NM_004451.5). Overexpression of ERRa or TFEB was achieved in KLE and ECC-1 cells and named KLE ${ }^{\text {TFEB-OV }}$, ECC-1 TFEB- OV , $\mathrm{KLE}^{\mathrm{ERRa}-\mathrm{OV}}$ and ECC-1 ${ }^{\mathrm{ERRa}-\mathrm{OV}}$, respectively. In addition, KLE and ECC-1 cells with ERRa or TFEB expression downregulated through lentivirus-mediated siRNA were named KLE ${ }^{\mathrm{TFEB}-K \mathrm{D}}, \mathrm{ECC}-1^{\mathrm{TFEB}-\mathrm{KD}}, \mathrm{KLE}^{\mathrm{ERRa}-\mathrm{KD}}$ and ECC-1 ${ }^{\mathrm{ERRa}-\mathrm{KD}}$, respectively.

\section{Bioinformatics Data Analyze}

Gene expression data (575 cases, workflow type: HTSeqCounts) were downloaded from The Cancer Genome Atlas (TCGA) official website for the Uterine Corpus Endometrial Carcinoma (UCEC) projects. The dataset included 23 normal endometrial specimens and 543 EC specimens, with 9 repeated cancerous specimens excluded. Patient clinical information, gene-level copy number variation (CNV) profiles, gistic2 thresholds analyzed by the GISTIC2.0 method and somatic nonsilent mutation (gene-level) data were acquired from the University of California, Santa Cruz (UCSC) Xena website. The Database for Annotation, Visualization and Integrated Discovery (DAVID) (version 6.8) provides a comprehensive set of functional annotation tools that help investigators understand the biological meaning behind a large list of genes. GO functional annotation and KEGG analysis of the DEGs were performed, and the results were visualized with the cluster Profiler R package.

\section{Rna Extraction, Rt-qpcr And Western Blotting (Wb)}

RNA extraction, RT-qPCR and Western blotting (WB)

Samples were collected from an equal number of intact cells in TRI Reagent ${ }^{\circledR}$ (\#TR118; Molecular Research Center). After reverse transcription on 500 ng of total RNA with RevertAid Reverse Transcriptase, oligo-dT and random hexamers (Thermo Fisher Scientific), quantitative PCR amplification was performed on a ViiA $^{\text {TM }} 7$ real-time PCR system (Applied Biosystems) using Takyon Low Rox SYBR® MasterMix dTTP Blue (\#UFLSMT-B0701; Eurogentec). Relative gene expression was calculated using the ddCt method, with GAPDH as the reference gene. Standard techniques were used for protein quantification, separation, transfer, and blotting. Primary antibodies against the following targets were used: TFEB (1:1000; Cell Signaling Technology, China), ERRa (1:500; Abcam, UK), LPCAT1 (1:1000; Proteintech, China), LPCAT3 (1:1000; Proteintech, China), MMP2 (1:1000; Beyotime Biotechnology, China), Cortactin (1:1000; Beyotime Biotechnology, China), E-cadherin (1:1000; Cell Signaling Technology, China), vimentin (1:1000; Cell Signaling Technology, China), MRPS2 (1:1000; Proteintech, China) and GAPDH (1:2000; Proteintech, China).

\section{Wound Healing}

Cells were grown to confluence in 6-well plates, and a $200-\mu \mathrm{L}$ tip was used to introduce a scratch in the monolayer. The scratch areas in the wells were washed with PBS and $1 \mathrm{mmol} / \mathrm{L}$ R-flurbiprofen until the cells in those areas were removed thoroughly and imaged at 0 and 24 hours postscratching. The horizontal migration rate was calculated using the following formula: (width $0 \mathrm{~h}$ - width $24 \mathrm{~h}$ )/ width $0 \mathrm{~h} \times 100 \%$.

\section{Chromatin-immunoprecipitation (Chip) Assay}

Cells were harvested followed by cross-linking for 10 min with $1 \%$ (vol/vol) formaldehyde. Afterwards, cells were lysed by sonication. The cell lysates were immunoprecipitated with anti-TFEB (ab2636, Abcam) overnight at $4^{\circ} \mathrm{C}$. After washing and elution, the crosslinks were reversed for 4 hours at $65^{\circ} \mathrm{C}$. The eluted DNA was purified and analyzed by qPCR using a Bio-Rad SYBR Green intercalating fluorophore system with the following ERRa primers: $5^{\prime}$-AGT TTA TGT GGC TGT GGG CA-3' (forward) and 5'-GGA TAT TTG CTG TCT TTA TAT TC-3' (reverse). The Ct value of each sample was normalized to the corresponding input value.

\section{Luciferase Reporter Assays}

Bioinformatics methods were used to analyze and predict the potential transcription factor binding sites in the ERRa promoter region. The ERRa promoter sequence ( 64303524 bp to 64305524 bp) relative to the transcription start site was amplified by PCR and inserted into the pGL3-basic vector (E1751, Promega). KLE cells were cotransfected with empty pcDNA3.1 vector or TFEB-S211A pcDNA3.1 plasmid in 24-well plates with Lipofectamine 2000. After 48 hours, the firefly and Renilla luciferase activities were measured using the Dual-Luciferase Reporter Assay Kit (E1901, Promega) and a microplate reader (Synergy H1, Bio-Tek), and the ratio of firefly/Renilla luciferase activity was determined. 


\section{Lipid And Metabolite Profiling}

Liquid Chromatography Mass Spectrometry (LC/MS) analyses were performed using a high-performance liquid chromatography system (1260 series; Agilent Technologies) and mass spectrometer (Agilent 6460; Agilent Technologies). Briefly, 107/ml EC cells or $20 \mathrm{mg}$ EC tissue was homogenized in $1.5 \mathrm{~mL}$ of chloroform/methanol $(2: 1, \mathrm{v} / \mathrm{v})$, vortexed for 1 minute, centrifuged at 3,000 rpm for 10 minutes, added to $800 \mu \mathrm{L}$ organic phase in a clean tube, and dried with nitrogen. Sample preparation processes were performed in accordance with the above method of parallel preparation of quality control samples. Mass spectrometric analysis was conducted by adding $200 \mu \mathrm{L}$ isopropanol/methanol solution $(1: 1, \mathrm{v}: \mathrm{v})$, and the supernatant was used for analysis. For targeted metabolomic analyses, multiple reaction monitoring transitions representing the metabolites were simultaneously monitored, and positive/negative polarity switching was used. Data analyses were performed according to the instructions of Shanghai Applied Protein Technology ${ }^{20}$.

\section{Tandem Mass Tag (Tmt) Labeling Proteomics}

The total protein in KLE cells and KLE-XCT790 cell samples was extracted and evaluated by SDS-PAGE and staining. The qualified protein samples were labeled with trypsin and TMT. The labeled polypeptides were mixed into one component in equal quantities. After desalination, high-pH RP-HPLC was used for grading. Eight different polypeptide components were obtained, and each component was separated by nano-HPLC and detected by mass spectrometry. Then, maxQuant search software was used for protein identification and quantitative analysis. After the quantitative results were standardized, statistical analysis was conducted to screen out the differentially expressed proteins.

\section{Mitochondrial Stress Detection}

Mitochondrial stress detection was conducted by using the Seahorse XF Cell Mitochondrial Stress Test Kit. First, prepare the test solution (Seahorse XF DMEM medium with additives including $1 \mathrm{mmol} / \mathrm{L}$ sodium pyruvate, $2 \mathrm{mmol} / \mathrm{L}$ glutamine, and $10 \mathrm{mmol} / \mathrm{L}$ glucose). The test solution was heated in a water bath to $37^{\circ} \mathrm{C}$ and prepared for use. Next, prepare the compound storage and working fluid. Using a Seahorse XF cell mitochondrial stress test kit, oligomycin (blue cap), $0.5 \mu \mathrm{M}$ FCCP (yellow cap), and rotenone/antimycin A (red cap) were properly prepared into a working solution and added to the dole on the probe plate. Seahorse XF cell culture microplates were removed from a $37^{\circ} \mathrm{C} \mathrm{CO}$ incubator, and the cells were examined under a microscope to confirm the degree of confluence. Remove the test solution from the water bath. The cell growth medium in the cell culture microplates was replaced with preheated detection solution using a multichannel pipette, and the cell culture microplates were placed in a $\mathrm{CO}_{2}$-free incubator at $37^{\circ} \mathrm{C}$ for 1 hour. Then, run the experiment on the computer and analyze the data.

\section{Immunohistochemistry (Ihc)}

All tissues were assembled into a tissue microarray. Immunostaining for ERRa and TFEB was performed according to standard procedures. Rabbit polyclonal anti-ERRa (dilution 1:100; Abcam, UK) and rabbit polyclonal anti-TFEB (dilution 1:100; Cell Signaling Technology, China) antibodies were used. The percentage of positive cells was scored as 0 (cells < 5\%), 1 (5-25\%), 2 (26-50\%), $3(51-75 \%)$, and 4 (76-100\%). The positive staining intensity was scored as 0 (no staining), 1 (weak staining), 2 (moderate staining), and 3 (strong staining). The expression levels of ERRa and TFEB were assessed to determine their immunoreactive scores (IRSs) using the algorithm IRS = Si $\times$ Pi (where Si and Pi represent the intensity and percentage of positively stained cells, respectively). Samples were divided into four groups based on their IRS: 0 , negative (-); $1-4$, weakly positive (+); $5-8$, positive (++); and 9-12, strongly positive (+++).

\section{Participants And Specimens}

EC tissue and normal endometrial tissue samples and blood samples with relevant clinical data were obtained from surgical patients in Fujian Provincial Maternity and Children's Health Hospital of Fujian Medical University, China from 2013 to 2018. None of the patients received any preoperative radiation, chemotherapy or hormone therapy. Finally, we collected 111 tissue specimens, including 79 EC specimens and 32 normal endometrium specimens. The samples were embedded in paraffin, and all diagnoses of the pathological sections were made by experienced pathologists. In addition, according to ERRa immunoreactive scores, 35 cases with the highest score and 20 cases with the lowest score were selected for lipidomic analysis. Finally, because of 1 EC tissue sample missed (IRS = 7), a total of 54 patient tissues from 35 EC patients and 19 patients with normal endometrium were also collected for lipidomics analysis (Supplement Fig. 1). All patients were informed of the experiments and signed informed consent forms. This research protocol was approved by the Ethics Committee of Fujian Provincial Maternity and Children's Hospital (No. FMCH-2018-14).

\section{Statistical analysis}

Statistical analysis was performed using GraphPad Prism 8.0 software and IBM SPSS (version 22). Statistical significance was determined by Student's t test or by ANOVA, and related parameters were analyzed using Pearson's correlation. Correlation coefficients for graded data were obtained using Pearson correlation analysis. Receiver operating characteristic (ROC) curves and the Youden Index were used to determine the cut-off point of continuous variable. The univariate binary logistic regression analyses was used to analyse indicators associated with EC. Differences with p-values less than 0.05 were considered significant.

\section{Results}




\section{Bioinformatics analysis revealed that TFEB promotes ERRa transcription to participate in EC progression}

To explore the role of TFEB and ERRa in EC, we first investigated the expression and clinicopathological data of these two genes in 543 EC samples and 23 normal samples from TCGA RNA-seq database. High expression of TFEB was significantly associated with a more advanced stage $(p=0.012$; Fig. $1 A)$ but not with pathological grade in EC ( $p=0.106$; Fig. 1B). Moreover, EC patients with high expression of TFEB had worse overall survival (OS) than those with low expression ( $p=0.037$; Fig. $1 \mathrm{C}$ ). Similarly, high ERRa expression was significantly associated not only with more advanced stages but also grades in EC (both $\mathrm{p} \otimes 0.05$; Fig. 1D-F). Consistent with our purpose, the expression of TFEB was significantly positively correlated with ERRa (Pearson coefficient $=0.168 ; \mathrm{p} \varangle 0.001$; Fig. 1G)

\section{ERRa is a direct transcriptional target of TFEB involved in lipid metabolism in EC}

Previously, we demonstrated that the transcriptional activity of TFEB correlated with ERRa, but the exact mechanism remains unclear. Luciferase activity detection showed that the relative luciferase activity triggered by ERRa expression was significantly enhanced by the promotion of TFEB (Fig. $1 \mathrm{H}$ ). To further study the crosstalk between TFEB and ERRa, ChIP-qPCR was performed, and the results confirmed that TFEB could directly bind to the promoter of ERRa DNA. Seven possible TFEB transcriptional binding sites (Fig. 1l; region P1-P7; all relative scores >0.80) on the promoter region of the ERRa gene were predicted. Among them, the P5 site with the element sequence 3'-CGCACGTGGC-5' was the most likely combination with TFEB (Fig. $1 \mathrm{~J}$ ). These data strongly indicate that TFEB could directly bind to the ERRa promoter and positively regulate ERRa expression. TFEB is the key regulator involved in lipid catabolism. Gene set enrichment analysis (GSEA) of the high- and low-ERRa expression groups was conducted to explore and identify the potential function of ERRa in EC. The gene sets with nominal $p$ value $<0.05$ and FDR $<0.25$ were considered significantly enriched in fatty acid (FA) metabolism and adipogenesis (Fig. $1 \mathrm{~K}$ ). There might be an important biological function, especially in the lipid metabolism pathway crosstalk with the expression of TFEB and ERRa.

\section{Erra Elevated Unsaturated Fatty Acid (Ufa)-containing Gps In Ec}

Subsequently, lipidomics was performed and analyzed in KLE ERRa-OV cells. In general, 7 categories of lipids, which were composed of 1120 glycerophospholipids (GPs), 345 sphingolipids (SPs), 285 glycerolipids (GLs) and other lipid categories, were screened and identified based on a liquid chromatography mass spectrometry (LC-MS)/MS system (Fig. 2A). Finally, 36 classes of lipids were tested, which included 395 phosphatidylcholines (PCs), 252 triacylglycerols (TAGs), 236 phosphatidylethanolamines (PEs) and other lipid species (Fig. 2B). Systematic lipidomic changes occurring between KLE and KLE ERRa-OV were then assessed by orthogonal partial least squares-discriminant analysis (OPLS-DA). There was obvious heterogeneity between the groups, with $R^{2} Y=0.989$ and $Q^{2}=0.838$ (Fig. 2C). Among them, digalactosyldiacylglycerol (DGDG) was observed to increase with fold change (FC) $>1.5$, while gangliosides2 (GM2) was reduced significantly with $\mathrm{FC}<0.67$. Although PC and sphingomyelin (SM) changed with ERRa elevation indistinctively, the PC/SM ratio was significantly increased in the ERRa-OV group, which was used to evaluate cell membrane fluidity $(P<0.05$, Fig. $2 D)$. This finding indicates that high expression of ERRa is likely to elevate EC cell membrane fluidity. After regulation by ERRa, 7 species of lipids, including PCs, phosphatidylglycerols (PGs), cardiolipins (CLs), PE (18:1/20:5), phosphatidylserines (PSs) and ceramides (Cers), with fold changes higher than 1.5 (VIP > 1 and $p<0.05)$ were observed. Increased UFA-containing PCs, PGs, PSs, SMs, CLs and PE (18:1/20:5) were found in KLE ${ }^{\text {ERR-OV }}$, while a decrease in saturated fatty acid (SFA)-containing PCs, PGs and SM (40:0) was also detected in KLE ${ }^{E R R-O V}$ (Fig. 2E). In brief, the common event is that UFA-containing GPs are increased in ERRa-overexpressing EC cells.

The data showed that the membrane fluidity of KLE was increased with ERRa. Hence, TFEB drives ERRa to elevate the unsaturation of fatty acyl moieties in GPs, which enhances membrane fluidity for invasion and metastasis.

\section{Proteins/lipids related to ERRa were enriched in mitochondrial function in EC}

Compared to control KLE cells, 173 proteins related to ERRa with unique peptides $\geq 2$, FC $>1.3$ and $p<1.0$ were gained in KLE cells treated with XCT790. The biological process of these proteins was mainly enriched in mitochondrial function, and the cell component was enriched in cone filopodium growth (Fig. $3 \mathrm{~A}$ ). Since an identified potential biological process was found to be affected by ERRa, the concentrations of these proteins/lipids were next evaluated in EC invasion and metastasis. 32 proteins were significantly different between UFA-containing PCs, PGs, SMs and SFA-containing PCs, PGs, and SMs by combining proteomics and lipidomics (Fig. 3B-C, Supplement Table 1). The oxidative phosphorylation (OXPHOS) pathway was enriched by KEGG analysis (Fig. 3D). Therefore, we routinely detected proteins associated with FA metabolism, including acc, fasn and acadm, which showed the FA metabolism was dynamic. All the proteins were upregulated as ERRa was increased and downregulated as ERRa decreased ( $p<0.05$ Fig. 3E). Subsequently, mitochondrial stress was evaluated by an energy analyzer. The maximum oxygen consumption rate (OCR) of cells treated with $0.5 \mu \mathrm{M}$ FCCP was increased by nearly $20 \%$ in KLE ${ }^{\text {TFEB-OV }}$ and KLE ${ }^{E R R a-O V}$ compared to that of their controls. Similarly, the OCRs of KLE ${ }^{\text {TFEB-KD }}$ and KLE ${ }^{\text {ERRa-KD }}$ cells were decreased by $50 \%$ and $40 \%$, respectively ( $p<$ 0.05 ; Fig. 3F). The same trend was observed in the acute response levels $(p<0.05)$. The level of ATP showed a positive correlation of TFEB and ERRa $(p<$ 0.05). Although ATP induced by KLE ERRa did not significantly increase, it still showed an upward trend $(p>0.05 ;$ Fig. $3 F)$. The degree of saturation of fatty acyl moieties of membrane phospholipids determines the biophysical properties of cell membranes, such as their fluidity. It is well known that LPCAT1 and LPCAT3 are the key phospholipid remodeling enzymes that regulate the degree of saturation of fatty acyl moieties in the membrane ${ }^{16}$. Thus, we tested the FA desaturase proteins LPCAT1 and LPCAT3. LPCAT1/3 were increased in KLEERR-OV compared with the controls. In contrast, both LPCAT1 and LPCAT3 decreased in KLE ERRa-KD compared with the controls $(p<0.05)$. Meanwhile, MMP2 and Cortactin were detected to evaluate membrane fluidity $(p<0.05$; 
Fig. 3G). These data suggested that TFEB could induce mitochondria stress and phospholipid remodeling to elevate the fluidity of the cell membrane by upregulating ERRa in KLE cells.

Table 1

The level of serum lipid, ERRa and TFEB in tissue microarray of EC patients and controls.

\begin{tabular}{|c|c|c|c|c|c|c|c|c|c|c|c|c|c|c|c|c|c|}
\hline \multirow{2}{*}{$\begin{array}{l}\text { Parameter } \\
(\mathrm{N}=111)\end{array}$} & \multirow[t]{2}{*}{ TG } & & \multirow[t]{2}{*}{$\mathrm{CHOL}$} & & \multirow[t]{2}{*}{$\mathrm{HDL}$} & & \multirow[t]{2}{*}{ APO-A } & & \multirow[t]{2}{*}{ LDL } & & \multirow[t]{2}{*}{ APO-B } & & \multicolumn{4}{|c|}{ TFEB } & \multirow[t]{2}{*}{$E$} \\
\hline & & & & & & & & & & & & & $-/+$ & ++ & +++ & & \\
\hline $\begin{array}{l}\text { Normal (n } \\
=32)\end{array}$ & $\begin{array}{l}1.027 \\
\pm \\
0.882\end{array}$ & 0.032 & $\begin{array}{l}4.623 \\
\pm \\
0.853\end{array}$ & 0.649 & $\begin{array}{l}1.599 \\
\pm \\
0.412\end{array}$ & 0.041 & $\begin{array}{l}1.331 \\
\pm \\
0.229\end{array}$ & 0.026 & $\begin{array}{l}2.709 \\
\pm \\
0.830\end{array}$ & 0.568 & $\begin{array}{l}0.834 \\
\pm \\
0.229\end{array}$ & 0.157 & 24 & 8 & 0 & $<0.001$ & 2 \\
\hline $\begin{array}{l}\text { EC } \\
(n=79)\end{array}$ & $\begin{array}{l}1.549 \\
\pm \\
1.369\end{array}$ & & $\begin{array}{l}4.723 \\
\pm \\
1.032\end{array}$ & & $\begin{array}{l}1.394 \\
\pm \\
0.428\end{array}$ & & $\begin{array}{l}1.207 \\
\pm \\
0.226\end{array}$ & & $\begin{array}{l}2.578 \\
\pm \\
0.929\end{array}$ & & $\begin{array}{l}0.924 \\
\pm \\
0.338\end{array}$ & & 26 & 38 & 15 & & 1 \\
\hline $\begin{array}{l}\text { Stage I-II } \\
(n=50)\end{array}$ & $\begin{array}{l}1.556 \\
\pm \\
1.538\end{array}$ & 0.859 & $\begin{array}{l}4.703 \\
\pm \\
1.133\end{array}$ & 0.192 & $\begin{array}{l}1.388 \\
\pm \\
0.470\end{array}$ & 0.980 & $\begin{array}{l}1.191 \\
\pm \\
0.224\end{array}$ & 0.691 & $\begin{array}{l}2.693 \\
\pm \\
0.985\end{array}$ & 0.178 & $\begin{array}{l}0.935 \\
\pm \\
0.381\end{array}$ & 0.785 & 19 & 31 & 12 & 0.710 & 1 \\
\hline $\begin{array}{l}\text { Stage III- } \\
\text { IV }(\mathrm{n}=12)\end{array}$ & $\begin{array}{l}1.622 \\
\pm \\
1.019\end{array}$ & & $\begin{array}{l}5.055 \\
\pm \\
0.719\end{array}$ & & $\begin{array}{l}1.386 \\
\pm \\
0.234\end{array}$ & & $\begin{array}{l}1.218 \\
\pm \\
0.210\end{array}$ & & $\begin{array}{l}3.148 \\
\pm \\
0.636\end{array}$ & & $\begin{array}{l}0.958 \\
\pm \\
0.236\end{array}$ & & 7 & 7 & 3 & & 1 \\
\hline $\begin{array}{l}M \mathrm{l}<50 \% \\
(\mathrm{n}=55)\end{array}$ & $\begin{array}{l}1.597 \\
\pm \\
1.531\end{array}$ & 0.589 & $\begin{array}{l}4.742 \\
\pm \\
1.064\end{array}$ & 0.806 & $\begin{array}{l}1.388 \\
\pm \\
0.463\end{array}$ & 0.827 & $\begin{array}{l}1.196 \\
\pm \\
0.219\end{array}$ & 0.550 & $\begin{array}{l}2.593 \\
\pm \\
0.979\end{array}$ & 0.880 & $\begin{array}{l}0.936 \\
\pm \\
0.362\end{array}$ & 0.618 & 21 & 29 & 5 & 0.003 & 1 \\
\hline $\begin{array}{l}M I \geq 50 \% \\
(n=25)\end{array}$ & $\begin{array}{l}1.445 \\
\pm \\
0.933\end{array}$ & & $\begin{array}{l}4.680 \\
\pm \\
0.979\end{array}$ & & $\begin{array}{l}1.408 \\
\pm \\
0.350\end{array}$ & & $\begin{array}{l}1.231 \\
\pm \\
0.244\end{array}$ & & $\begin{array}{l}2.553 \\
\pm \\
0.869\end{array}$ & & $\begin{array}{l}0.898 \\
\pm \\
0.282\end{array}$ & & 5 & 9 & 10 & & 0 \\
\hline $\begin{array}{l}\text { EEC } \\
(n=64)\end{array}$ & $\begin{array}{l}1.515 \\
\pm \\
1.397\end{array}$ & 0.592 & $\begin{array}{l}4.790 \\
\pm \\
1.056\end{array}$ & 0.184 & $\begin{array}{l}1.424 \\
\pm \\
0.452\end{array}$ & 0.120 & $\begin{array}{l}1.218 \\
\pm \\
0.225\end{array}$ & 0.390 & $\begin{array}{l}2.706 \\
\pm \\
0.976\end{array}$ & 0.021 & $\begin{array}{l}0.950 \\
\pm \\
0.353\end{array}$ & 0.100 & 20 & 32 & 11 & 0.613 & 1 \\
\hline $\begin{array}{l}\text { NEEC }(n= \\
16)\end{array}$ & $\begin{array}{l}1.711 \\
\pm \\
1.256\end{array}$ & & $\begin{array}{l}4.412 \\
\pm \\
0.887\end{array}$ & & $\begin{array}{l}1.274 \\
\pm \\
0.301\end{array}$ & & $\begin{array}{l}1.162 \\
\pm \\
0.232\end{array}$ & & $\begin{array}{l}2.133 \\
\pm \\
0.578\end{array}$ & & $\begin{array}{l}0.819 \\
\pm \\
0.254\end{array}$ & & 6 & 6 & 4 & & 1 \\
\hline $\begin{array}{l}\text { LNM } \\
(\mathrm{n}=9)\end{array}$ & $\begin{array}{l}1.605 \\
\pm \\
1.447\end{array}$ & 0.316 & $\begin{array}{l}4.724 \\
\pm \\
1.059\end{array}$ & 0.729 & $\begin{array}{l}1.367 \\
\pm \\
0.421\end{array}$ & 0.046 & $\begin{array}{l}1.193 \\
\pm \\
0.223\end{array}$ & 0.151 & $\begin{array}{l}2.640 \\
\pm \\
0.946\end{array}$ & 0.092 & $\begin{array}{l}0.925 \\
\pm \\
0.351\end{array}$ & 0.984 & 5 & 2 & 2 & 0.232 & 1 \\
\hline $\begin{array}{l}\text { No-LNM } \\
(\mathrm{n}=70)\end{array}$ & $\begin{array}{l}1.413 \\
\pm \\
0.380\end{array}$ & & $\begin{array}{l}4.836 \\
\pm \\
0.812\end{array}$ & & $\begin{array}{l}1.637 \\
\pm \\
0.439\end{array}$ & & $\begin{array}{l}1.326 \\
\pm \\
0.242\end{array}$ & & $\begin{array}{l}1.980 \\
\pm \\
0.666\end{array}$ & & $\begin{array}{l}0.923 \\
\pm \\
0.253\end{array}$ & & 21 & 35 & 13 & & 1 \\
\hline
\end{tabular}

Abbreviations: APO-A, A polipoprotein A. APO-B, A polipoprotein B. CHOL, Cholesterol. ERRa, Estrogen-Related Receptor a. EC, Endometrial cancer. ECC, Endor Adenocarcinoma. HDL, High-Density Lipoprotein. LDL, Low-Density Lipoprotein. LNM, Lymph Node Metastasis. MI, Myometrial invasion. NECC, Non-endomet TG, Total Triglyceride. TFEB, transcription factor EB. $P<0.05$ suggests significantly different.

TFEB promotes EC migration depending on ERRa via EMT signaling

Although the inhibitory effects of ERRa on EC invasion and metastasis have been established ${ }^{9}$, its underlying mechanisms are far from elucidated. The migration ability of KLE and ECC-1 cells was remarkably changed after regulation of the expression of TFEB or ERRa through a lentivirus-mediated strategy. Compared to the controls, the scratched spaces were up to $47.2 \%$ in $\mathrm{KLE}^{\mathrm{TFEB}-\mathrm{OV}}, 21.9 \%$ in $\mathrm{KLE}^{\mathrm{ERRa}-\mathrm{OV}}$ at $24 \mathrm{~h}$ and up to $126.4 \%$ in ECC-1 ${ }^{\mathrm{TFEB}-\mathrm{OV}}$, respectively (both $p<0.05$; Fig. 4A-B). The scratched spaces of ECC- 1 ERRa-OV at $24 \mathrm{~h}$ also increased slightly, but no significant difference from their controls ( $p>0.05$ ). The wounded spaces were nearly 2-fold decreased at $24 \mathrm{~h}$ in both $\mathrm{KLE}^{\mathrm{TFEB}-K D}$ and KLE ERRa-KD cells compared with their controls ( $\left.p<0.001\right)$. A similar trend was observed in ECC-1 ${ }^{\text {TFEB }-K D}$ and ECC-1 ${ }^{\text {ERRa-KD }}$. To further confirm the effects of TFEB on ERRa-mediated cell migration, ERRa levels were inhibited in KLE ${ }^{\text {TFEB-OV }}$

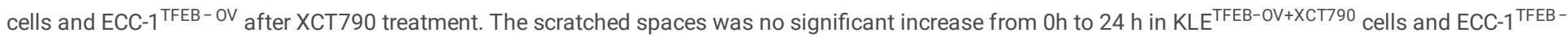
$\mathrm{OV}^{\mathrm{XCCT790}}$. Meanwhile, the enhanced migration abilities of KLE ${ }^{\mathrm{TFEB}-\mathrm{OV}}$ and ECC-1 ${ }^{\text {TFEB-OV }}$ cells were partially compromised after XCT790 treatment $(p<0.05$;

Fig. 4A-B). Moreover, the qPCR results showed that downregulation of TFEB reduced the expression of ERRa and Vimentin and increased the expression of Ecadherin in ECC-1 and KLE cells. In contrast, TFEB overexpression enhanced the expression of ERRa and Vimentin and decreased the expression of E-cadherin $(p<0.05$, Fig. $4 \mathrm{C})$. These data are similar to the findings observed in western blot experiments $(\mathrm{p}<0.05$, Fig. $4 \mathrm{E}-\mathrm{F})$. Importantly, there was no significant change in ERRa, Vimentin or E-cadherin expression when $\mathrm{KLE}^{\mathrm{TFEB}-\mathrm{OV}}$ cells were treated with $10 \mu \mathrm{M} X \mathrm{XT} 790$ ( $p>0.05$, Fig. 4F). This demonstrated that TFEB could regulate cell migration in an ERRa-dependent manner via the EMT signaling pathway.

\section{High expression of TFEB-ERRa is associated with dyslipidemia and metastasis in EC patients}


To verify the results obtained above, IHC was performed on an EC tissue microarray (TMA), which included 79 EC specimens and 32 normal endometrium specimens. Positive immunoreactivity for TFEB was detected in the nuclei of both carcinoma cells and normal endometrial gland cells. Significantly higher immunoreactivity was observed in EC tissue than in normal endometrial tissue ( $p<0.001$; Fig. 5A \& Table 1). Similarly, ERRa could also be detected in 79 of 79 (100\%) EC tissue samples with a higher immunoreactivity than it detected in 30 of 32 (93.75\%) normal endometrial tissues ( $P<0.001 ;$ Fig. $5 A$ \& Table 1$)$. There was no significant difference in TFEB or ERRa expression among EC patients with different International Federation International of Gynecology and Obstetrics (FIGO) stages, histologic tumor grades, pathological types or lymph node metastasis (LNM) conditions ( $p>0.05$; Fig. 5C). However, significant differences were detected between the $\leq 1 / 2$ and $>1 / 2$ myometrial invasion (MI) groups for both TFEB and ERRa expression in EC patients $(P<0.05$; Fig. $5 C$ ). Moreover, a positive correlation between TFEB and ERRa immunoreactivity was found based on Pearson's rank correlation analysis $(r=0.642$, $p<0.001$, Fig. 5B).

Interestingly, in a study on the same population for serum lipids, the serum total triglyceride (TG) level was significantly higher, while the high-density lipoprotein (HDL) and apolipoprotein A (APO-A) levels were significantly lower in EC patients than in normal people $(P<0.05$, Table 1$)$. In addition, the lowdensity lipoprotein (LDL) level was obviously higher in patients with endometrioid adenocarcinoma (EEC) than in patients with non-endometrioid adenocarcinoma (NEEC). Importantly, both HDL and APO-A levels were decreased significantly in patients with LNM ( $p<0.05$, Table 1). Next, we compared the differences in serum lipids in populations with different TFEB/ERRa expression levels. Not unexpectedly, serum HDL and APO-A levels were much lower in the patients with high expression of TFEB and ERRa $(++/+++)$ than in those with low TFEB and ERRa expression $(-/+)(p<0.05$; Fig. 5D). All this evidence suggests that elevated TFEB/ERRa is involved in EC invasion and metastasis and is related to decreases in serum HDL and APO-A levels.

\section{Accumulation of UFA-containing GPs induced by ERRa is required for EC progression}

The species of lipids obtained from EC tissues and normal endometrial tissues (patients $=35$ vs controls $=19$ ) were similar to the cells, which included 359 PCs, 324 TAGs, 269 PEs and other lipid species (Fig. 6A-B). Systematic lipidomic changes occurring between EC patients and normal controls were assessed by OPLS-DA. There was obvious heterogeneity between the populations, with $R^{2} Y=0.963$ and $Q^{2}=0.510$ (Fig. $6 C$ ). Fair discrimination was also found between patients with LNM and those without LNM. $\left(R^{2} Y=0.789\right.$ and $Q^{2}=0.574$, Fig. $\left.6 D\right)$. Consistent with the results from in vitro research in cells, $D G D G$ was observed to increase with fold change (FC) >1.5. Besides, PGs including phosphatidylinositol (PI), lysophosphatidylserine (LPS),

Lysophosphatidylethanolamine (LPE) and PG, Sphingolipids (SLs) including Hex2Cer, Hex1Cer, Cer and ceramides phosphate (CerP), GLs including monoglyceride (MG) and TG were also increased in EC tissue (Fig. 6E). Although SM elevated indistinctively, the PC/SM ratio was significantly increased in EC tissue, which was used to evaluate cell membrane fluidity $(\mathrm{P}<0.05$, Fig. 6E). In addition, UFA-containing GPs, such as PC (18:1/18:2) + HCO0, PC (32:2) + H, PG $(18: 1 / 22: 6)+\mathrm{H}$ and SM (42:1) + HCOO, were obviously increased in EC tissue compared to normal endometrium (FC $>1.5, \mathrm{VIP}>1$ and $\mathrm{p}<0.05 ;$ Fig. $6 \mathrm{~F})$. Moreover, compared to patients without LNM, PC (18:1/18:2) + HCOO was much higher in the patients with LNM (p<0.05; Fig. 6F). In addition, we performed the ROC curve analysis, and obtained the cut-off values. The results showed that PC (18:1/18:2) + HCOO and CA125 have good diagnostic value in patients with advanced stage EC ( $p<0.05$; Supplement Fig. 2). Moreover, PC (18:1/18:2) + HCOO and PC (32:2) + H were found to be independent risk factors for EC patients in advanced stage by the univariate binary logistic regression analyses $(P<0.05$, Fig. $6 G)$. Furthermore, we compared the differences in serum lipids in populations with different expression PC (18:1/18:2) + HCOO levels. The results showed serum HDL level was much lower in the patients with higher expression of PC (18:1/18:2) + HCOO than in those with lower PC (18:1/18:2) + HCOO $(p<0.05$, Table 2). Moreover, a positive correlation between PC (18:1/18:2) + HCOO and ERRa immunoreactivity was found based on Pearson's rank correlation analysis $(r=0.805, p<0.001$, Fig. $6 \mathrm{H})$. These results suggested that increased PC (18:1/18:2) + HCOO induced by ERRa was a novel biomarker of EC progress, which was probably related to the decrease of serum HDL.

Table 2

Associations of PC(18:1/18:2) + HCOO with serum lipid.

\begin{tabular}{|c|c|c|c|}
\hline Parameter & $\begin{array}{l}P C(18: 1 / 18: 2)+H C O O<\text { cutoff value } \\
(n=24)\end{array}$ & $\begin{array}{l}P C(18: 1 / 18: 2)+H C O O \geq \text { cutoff value } \\
(n=28)\end{array}$ & $P$ \\
\hline $\mathrm{TG}(\mathrm{mmol} / \mathrm{L})$ & $1.533 \pm 0.650$ & $2.911 \pm 9.981$ & 0.136 \\
\hline $\mathrm{CHOL}(\mathrm{mmol} / \mathrm{L})$ & $5.379 \pm 1.062$ & $5.329 \pm 1.258$ & 0.904 \\
\hline $\mathrm{HDL}(\mathrm{mmol} / \mathrm{L})$ & $1.416 \pm 0.345$ & $1.190 \pm 0.313$ & 0.028 \\
\hline APO-A(g/L) & $1.083 \pm 0.130$ & $1.057 \pm 0.182$ & 0.645 \\
\hline $\mathrm{LDL}(\mathrm{mmol} / \mathrm{L})$ & $3.367 \pm 0.958$ & $2.960 \pm 0.757$ & 0.221 \\
\hline APO-B(g/L) & $1.011 \pm 0.261$ & $0.961 \pm 0.200$ & 0.573 \\
\hline \multicolumn{4}{|c|}{ Abbreviations: APO-A, A polipoprotein A. APO-B, A polipoprotein B. CHOL, Cholesterol. } \\
\hline
\end{tabular}

In conclusion, TFEB-ERRa axis promotes the UFA-containing GPs accumulation to induce lipid reprogramming hallmarked by mitochondrial stress, which contributes to the invasion and metastasis of EC (Supplement Figure.3). Importantly, the UFA-containing PC, SM and PG were the significantly altered lipids founded in EC cells and tissues, among which, PC (18:1/18:2) + HCOO was the ERRa-associated potential predictor of EC metastasis. 


\section{Discussion}

EC is one of the cancers most related to metabolic disorders, and patients present with hyperlipidemia, hyperglycemia, hypertension and other clinical symptoms ${ }^{21}$. Guo et al suggested that metformin significantly reversed obesity-driven lipid and protein biosynthesis upregulation in an obese LKB ${ }^{\mathrm{fl} / \mathrm{fl}} \mathrm{p} 53^{\mathrm{fl} / \mathrm{fl}}$ mouse model of $\mathrm{EC}^{22}$. Recently, an increasing number of studies have confirmed that ERRa is a key regulator of metabolism in obesity-related tumors, such as breast cancer ${ }^{23}$, prostate cancer ${ }^{24}$, and $\mathrm{EC}^{25}$. Moreover, ERRa-mediated signaling pathways have recently emerged as key factors in the regulation of cancer lipid metabolism. In our previous work, the translational factor activity of TFEB was affected by the downregulation of ERRa expression in EC according to a high-throughput DNA/protein assay ${ }^{25}$, which suggested that TFEB should interact with ERRa and be involved in EC lipid reprogramming and progression, which triggered our interest. TFEB downregulation or deficiency can obviously affect the cellular phenotype in a physiologically relevant manner in settings including atherosclerosis ${ }^{26}$, nonalcoholic fatty liver disease ${ }^{27}$, cancer ${ }^{28}$ and neurodegeneration ${ }^{29}$. Furthermore, TFEB is activated by starvation or caloric restriction and plays roles in lipid catabolism and lysosomal biogenesis ${ }^{14}$. Therefore, we started with bioinformatics analysis of the TCGA data. In agreement with our hypothesis, the results confirmed that both TFEB and ERRa are strongly associated with a poor prognosis in EC, as reflected by their associations with a high FIGO stage and a shortened survival time. Moreover, the expression of TFEB was first found to be positively correlated with the expression of ERRa in the TCGA data of 543 EC cases. The bioinformatics analysis result was further verified by our clinical data from our TMA, in which TFEB and ERRa showed a strong correlation and both were related to the MI of EC. However, the exact interaction mechanism between TFEB and ERRa has not yet been described clearly. In 2019, TFEB was reported to drive PGC-1a expression in adipocytes to protect against diet-induced metabolic dysfunction, while PGC-1a is one of the most important coactivators of ERRa ${ }^{30}$. We further confirmed that TFEB can bind to the promoter region of ERRa and regulate the expression and function of ERRa in vitro by Chip assay and luciferase assay.

Previously, we have reported that high expression of ERRa is associated with cancer cell metastasis and invasion ${ }^{11}$. To our knowledge, this is the first report that TFEB takes part in the invasion of EC cells by EMT signaling. Interestingly, the patient's clinical lipid profile indicated that the serum HDL and APO-A was negatively correlated with TFEB and ERRa. And lower serum HDL and APO-A levels were associated with the LNM in EC patients. As we know, PC was the major phospholipids of HDL ${ }^{31}$. Importantly, we found there was a significant decrease of HDL in the patients with higher PC (18:1/18:2) + HCOO which indicated PC remodeling influenced the serum HDL level of EC patients probably. Together, TFEB/ERRa showed an early predictor of MI, while lower HDL/APOA and higher PC (18:1/18:2) + HCOO played the risk factors role of LNM in advanced EC. In brief, TFEB/ERRa regulates EC patients' lipid metabolism and is involved in the EC invasion and metastasis.

Moreover, our in vitro and in vivo lipidomics experiments first investigated ERRa as a downstream signal by which TFEB promotes UFA-GPs accumulation during EC progression. Guo found dramatic increases in lipid biosynthesis and lipid peroxidation in a genetically engineered mouse model of endometrioid adenocarcinoma ${ }^{22}$, suggesting that lipidomic changes or reprogramming are significant in EC. Previous studies have confirmed that downregulation of ERRa provides a potential therapeutic strategy and inhibits cellular metastasis and invasion in EC ${ }^{11,25}$. However, the mechanism by which ERRa regulates FA and GP synthesis was unexplored prior to our study. After overexpressing ERRa, the top three categories of lipids obtained were GPs, SPs and GLs. Among them, PC $(18: 1 / 18: 2)+\mathrm{HCOO}, \mathrm{PC}(32: 2)+\mathrm{H}, \mathrm{PG}(18: 1 / 22: 6)+\mathrm{H}$ and SM (42:1) + HCOO were found to be the most significantly increased lipids and were related to ERRa overexpression. These evidences indicated an unexpected role of ERRa in the FAs unsaturated and phospholipid remodeling pathways that are controlled by the rate-limiting metabolic enzymes including LPCAT1 and LPCAT3 ${ }^{32,33}$. The impacts of UFA-containing GPs accumulation on membrane fluidity have been proposed as secondary effects linked to desaturation induced by LPCAT1 and LPCAT3. The sensitivity of lipids to oxidative stress depends on their fatty acid moiety. Double bonds in MUFAs and PUFAs show a prevalent cis conformation, which produces bends and limits their rigid packing ${ }^{34}$. Since double bonds make fatty acyl chains more susceptible to oxidative stress, increased UFA-GPs promotes the membrane fluidity when ATP is elevating in this study. Commonly cancer cells possess different and complementary metabolic profile, microenvironment and adopting behaviors to generate more ATPs to fulfill the requirement of high energy that is further utilized in the production of proteins and other essentials required for cell survival, growth, and proliferation.

Mitochondria is partially autonomous organelles that depend on the import of certain proteins and lipids to maintain cell survival and membrane formation ${ }^{35}$. CL and/or PG are considered as mitochondria-specific phospholipids ${ }^{36}$. PG (18:1/22:6) was found to be increased in MYC-induced T cell acute lymphoblastic leukemia, renal cell carcinoma, hepatocellular carcinoma, and lung carcinoma ${ }^{37}$. Our results that PG $(18: 1 / 22: 6)+\mathrm{H}$ were much higher in EC patients well demonstrated the important role of mitochondria in maintaining membrane homeostasis. Synthesis of mitochondrial ATP plays a key role in inducing membrane curvature to establish cristae in eukaryotes ${ }^{38}$. The effect of mitochondrial stress was considered as the hallmark of membrane remodeling here, which demonstrated that ERRa did enhance membrane fluidity by stimulate mitochondrial to prepare for invasion and metastasis. However, mitochondria lack PC synthesizing enzymes, this lipid has to be imported from other organelles, such as endoplasmic reticulum (ER). The prevailing view is that a significant pool of cellular PC can also made de novo from PS in a pathway that originates in the ER and passes into and out of the mitochondrion ${ }^{39}$. The genes related with FA metabolism such as acc, fasn and acadm increased with ERRa, which suggested ERRa mobilized $\beta$-oxidation and De novo lipogenesis to facilitate lipid reprogramming. Besides, increased PC (18:1/18:2) + HCOO has been shown to be a good predictor for prostate cancer ${ }^{40}$. In line with these findings, we found the PC (18:1/18:2) + HCOO was not only related to EC but also associated with EC metastasis, which showed a potential role of tumor marker.

In summary, we found that the TFEB-ERRa signaling pathway regulates the invasion and metastasis of endometrial cancer cells through the EMT pathway and cell membrane fluidity. This regulation depends on ERRa to participate in the metabolism of lipids and cellular membrane remodeling. ERRa enhances UFA-PCs, PG (18:1/22:6) + H and SM (42:1) + HCOO in EC patients to promote cellular fluidity and result in invasion and metastasis. Furthermore, $\mathrm{PC}(18: 1 / 18: 2)+\mathrm{HCOO}$ is the ERRa-associated potential predictor of EC metastasis. This also explains why ERRa, as a key factor in energy metabolism, is a poor prognostic factor for EC.

\section{Abbreviations}


ACADM

Medium-chain Acyl-coenzyme A Dehydrogenase; ACC:Acetyl-Co A Carboxylase; APO-A:a polipoprotein A; ATCC:American Type Culture Collection; ATP:adenosine triphosphate; BMI:Body Mass Index; Cer:Ceramide; CerP:ceramides phosphate; Chip-qPCR:Chromatin Immunoprecipitation Quantitative Polymerase Chain Reaction; $\mathrm{CHOL}$ :Cholesterol; $\mathrm{CL}$ :Cardiolipin; $\mathrm{CNV}$ :Copy Number Variation; $\mathrm{CO}_{2}$ :Carbon Dioxide; DAVID:The Database for Annotation, Visualization and Integrated Discovery; DGDG:digalactosyldiacylglycerol; DMEM:Dulbecco's modification of Eagle's medium Dulbecco; DMSO:Dimethyl Sulfoxide; DNA:Deoxyribonucleic Acid; EC:Endometrial Cancer; EEC:Endometrioid Adenocarcinoma; EMT:Epithelial-Mesenchymal Transformation; ER:Endoplasmic Reticulum; ERa:estrogen receptor a; ERRa/ NR3B1/ESRRA:Estrogen-Related Receptor a; FA:Fatty Acid; FASN:Fatty Acid Synthase; FBS:Fetal Bovine Serum; FC:Fold Change; FCCP:Carbonyl cyanide 4-(trifluoromethoxy)phenylhydrazone; FIGO:Federation International of Gynecology and Obstetrics; GL:Glycerolipid; GM2:Gangliosides2; GP:Glycerophospholipid; GSEA:Gene Set Enrichment Analysis; HDL:High-Density Lipoprotein; HPLC:High Pressure Liquid Chromatography; IHC:Immunohistochemistry; IRS:Immunoreactive Score; KEGG:Kyoto Encyclopedia of Genes and Genomes; LC/MS:Liquid Chromatography Mass Spectrometry; LDL:Low-Density Lipoprotein; LNM:Lymph Node Metastasis; LPCAT:Lysophosphatidylcholine Acyltransferase; LPS:lysophosphatidylserine; LPE:Lysophosphatidylethanolamine; MG:monoglyceride; MI:Myometrial Invasion; MMP2:Matrix Metalloproteinase 2; MRPS2:Mitochondrial Ribosomal Proteins 2; NEEC:Non-Endometrioid Adenocarcinoma; OCR:oxygen consumption rate; OPLS-DA:Orthogonal Partial Least Squares-Discriminant Analysis; OS:Overall Survival; OXPHOS:Oxidative Phosphorylation; PBS:Phosphate Buffered Solution; PC:Phosphatidylcholine; PE:Phosphatidylethanolamine; PG:Phosphatidylglycerol; PI:phosphatidylinositol; PGC-1:Peroxisome Proliferator-Activated Receptor Coactivator-1; PPAR:Peroxisome Proliferator-Activated Receptor; PS:Phosphatidylserine; RNA:Ribonucleic acid; RT-qPCR:Reverse Transcription Polymerase Chain Reaction; ROC:Receiver operating characteristic; SFA:Saturated Fatty Acid; siRNA:Small Interfering RNA; SLs:Sphingolipids; SM:Sphingomyelin; TAG:Triacylglycerol; TCGA:The Cancer Genome Atlas; TFEB:transcription factor EB; TG:Total Triglyceride; TMA:Tissue Microarray; TMT:Tandem Mass Tags; UCEC:Uterine Corpus Endometrial Carcinoma; UCSC:University of California, Santa Cruz; UFA:Unsaturated Fatty Acid; WB:Western blotting.

\section{Declarations}

\section{Ethics approval and consent to participate}

This research protocol was approved by the Ethics Committee of Fujian Provincial Maternity and Children's Hospital (No. FMCH-2018-14).

\section{Consent for publication}

Not applicable

\section{Availability of data and materials}

The datasets analysed during the current study are available from the corresponding author on reasonable request.

\section{Competing interests}

The authors declare that they have no competing interests.

\section{Funding}

This work was supported by grants from the Fujian Provincial Nature Science Foundation of China (Grant no. 2017Y9062, 2017J01233, 2020J02059) and National Nature Science Foundation of China (Grant no.82002756).

\section{Author Contributions}

XiaoDan Mao designed the experiments and wrote the paper. Huifang Lei, Tianjin Yi conducted the partial experiments, data analysis and fund support. Pingping Su and ShuTing Tang conducted the experiments and contributed to the analysis of data. Binhua Dong and Guanyu Ruan contributed to the methods and performed the laboratory analyses, and provided valuable discussion. Alexander Mustea and Jalid Sehouli contributed to critically revised the article for important intellectual content. PengMing Sun contributed to the acquisition of data, critically revised the article for important intellectual content, and supervised the study. All authors gave their final approval of the version to be submitted.

\section{Acknowledgments}

Thanks are due to Central Laboratory, Fujian Medical University Union Hospital for assistance with the experiments and to Zhenli Li, Lili Chen, Meimei Huang and Tingting Jiang for their assistance with the methods and literature retrieval.

\section{References}

1. Swinburn, B. A. et al. The Global Syndemic of Obesity, Undernutrition, and Climate Change: The Lancet Commission report. Lancet 393, 791-846 (2019).

2. Sung, H. et al. Global patterns in excess body weight and the associated cancer burden. CA Cancer J Clin 69, 88-112 (2019).

3. Siegel, R. L., Miller, K. D., Fuchs, H. E. \& Jemal, A. Cancer Statistics, 2021. CA Cancer J Clin 71, 7-33 (2021).

4. Chen, W. et al. Cancer statistics in China, 2015. CA Cancer J Clin 66, 115-132 (2016).

5. Giguère, V., Yang, N., Segui, P. \& Evans, R. M. Identification of a new class of steroid hormone receptors. Nature 331, 91-94 (1988).

6. Deblois, G., St-Pierre, J. \& Giguère, V. The PGC-1/ERR signaling axis in cancer. Oncogene 32, 3483-3490 (2013). 
7. De Vitto, H. et al. Estrogen-related receptor alpha directly binds to p53 and cooperatively controls colon cancer growth through the regulation of mitochondrial biogenesis and function. Cancer Metab 8, 28 (2020).

8. Deblois, G. \& Giguère, V. Oestrogen-related receptors in breast cancer: control of cellular metabolism and beyond. Nat Rev Cancer 13, 27-36 (2013).

9. Valcarcel-Jimenez, L. et al. PGC1a Suppresses Prostate Cancer Cell Invasion through ERRa Transcriptional Control. Cancer Res 79, 6153-6165 (2019).

10. Sun, P. et al. Expression of estrogen receptor-related receptors, a subfamily of orphan nuclear receptors, as new tumor biomarkers in ovarian cancer cells. J Mol Med (Berl) 83, 457-467 (2005).

11. Chen, L. et al. PGC-1a and ERRa in patients with endometrial cancer: a translational study for predicting myometrial invasion. Aging (Albany NY) 12 , 16963-16980 (2020).

12. Sun, P. et al. Novel endocrine therapeutic strategy in endometrial carcinoma targeting estrogen-related receptor a by XCT790 and siRNA. Cancer Manag Res 10, 2521-2535 (2018).

13. Zhitomirsky, B. \& Assaraf, Y. G. Lysosomes as mediators of drug resistance in cancer. Drug Resist Updat 24, 23-33 (2016).

14. Settembre, C. et al. TFEB controls cellular lipid metabolism through a starvation-induced autoregulatory loop. Nat Cell Biol 15, 647-658 (2013).

15. Wu, Y. et al. Phospholipid remodeling is critical for stem cell pluripotency by facilitating mesenchymal-to-epithelial transition. Sci Adv 5, eaax7525 (2019).

16. Wang, B. \& Tontonoz, P. Phospholipid Remodeling in Physiology and Disease. Annu Rev Physiol 81, 165-188 (2019).

17. van Meer, G., Voelker, D. R. \& Feigenson, G. W. Membrane lipids: where they are and how they behave. Nat Rev Mol Cell Biol 9, 112-124 (2008).

18. Bi, J. et al. Oncogene Amplification in Growth Factor Signaling Pathways Renders Cancers Dependent on Membrane Lipid Remodeling. Cell Metab 30, 525-538.e528 (2019).

19. Lin, L. et al. Functional lipidomics: Palmitic acid impairs hepatocellular carcinoma development by modulating membrane fluidity and glucose metabolism. Hepatology 66, 432-448 (2017).

20. Wang, C. et al. Hepatocellular Carcinoma-Associated Protein TD26 Interacts and Enhances Sterol Regulatory Element-Binding Protein 1 Activity to Promote Tumor Cell Proliferation and Growth. Hepatology 68, 1833-1850 (2018).

21. Smith, D. C., Prentice, R., Thompson, D. J. \& Herrmann, W. L. Association of exogenous estrogen and endometrial carcinoma. N Engl J Med 293, 11641167 (1975)

22. Guo, H. et al. Reversal of obesity-driven aggressiveness of endometrial cancer by metformin. Am J Cancer Res 9, 2170-2193 (2019).

23. Deblois, G. et al. ERRa mediates metabolic adaptations driving lapatinib resistance in breast cancer. Nat Commun 7, 12156 (2016).

24. Wallace, M. \& Metallo, C. M. PGC1a drives a metabolic block on prostate cancer progression. Nat Cell Biol 18, 589-590 (2016).

25. Mao, X. et al. Dual targeting of estrogen receptor a and estrogen-related receptor a: a novel endocrine therapy for endometrial cancer. Onco Targets Ther 12, 6757-6767 (2019).

26. You, Y. et al. Sorting Nexin 10 Mediates Metabolic Reprogramming of Macrophages in Atherosclerosis Through the Lyn-Dependent TFEB Signaling Pathway. Circulation research 127, 534-549 (2020).

27. Zhang, Z. et al. The unfolded protein response regulates hepatic autophagy by sXBP1-mediated activation of TFEB. Autophagy, 1-15 (2020).

28. Zhang, C. et al. TFEB mediates immune evasion and resistance to mTOR inhibition of renal cell carcinoma via induction of PD-L1. Clin Cancer Res 25, 6827-6838 (2019).

29. Martini-Stoica, H., Xu, Y., Ballabio, A. \& Zheng, H. The Autophagy-Lysosomal Pathway in Neurodegeneration: A TFEB Perspective. Trends Neurosci 39, 221234 (2016).

30. Evans, T. D. et al. TFEB drives PGC-1a expression in adipocytes to protect against diet-induced metabolic dysfunction. Sci Signal 12 (2019).

31. A. Jonas, M.C. Phillips, Lipoprotein structure, in: D.E. Vance, J.E. Vance (Eds.), Biochemistry of Lipids, Lipoproteins and Membranes, 5th Edition, Elsevier, sterdam, 2008, pp. 485-506.

32. Swinnen, J. V., Dehairs, J. \& Talebi, A. Membrane Lipid Remodeling Takes Center Stage in Growth Factor Receptor-Driven Cancer Development. Cell Metab 30, 407-408 (2019).

33. Wang, B. et al. Phospholipid Remodeling and Cholesterol Availability Regulate Intestinal Stemness and Tumorigenesis. Cell Stem Cell 22, 206-220.e204 (2018).

34. J A Plumb, W Luo, D J Kerr. Effect of polyunsaturated fatty acids on the drug sensitivity of human tumour cell lines resistant to either cisplatin or doxorubicin. British Journal of Cancer. 67(4):728-33 (1993).

35. Flis, V. V. \& Daum, G. Lipid transport between the endoplasmic reticulum and mitochondria. Cold Spring Harb Perspect Biol. 5(6):a013235 (2013).

36. Zinser, E. et al. Phospholipid synthesis and lipid composition of subcellular membranes in the unicellular eukaryote Saccharomyces cerevisiae. J Bacteriol 173, 2026-2034 (1991).

37. Gouw, A. M. et al. The MYC Oncogene Cooperates with Sterol-Regulated Element-Binding Protein to Regulate Lipogenesis Essential for Neoplastic Growth. Cell Metab, 30, 556-572.e555 (2019).

38. Alexander Mühleip. et al. ATP synthase hexamer assemblies shape cristae of Toxoplasma mitochondria. Nat Commun. 12(1):120 (2021).

39. Friedman, J. R. et al. Lipid Homeostasis Is Maintained by Dual Targeting of the Mitochondrial PE Biosynthesis Enzyme to the ER. Dev Cell, 44, 261270.e266 (2018).

40. Buszewska-Forajta, M. et al. Lipidomics as a Diagnostic Tool for Prostate Cancer. Cancers (Basel), 13: 2000 (2021). 


\section{Figures}

A

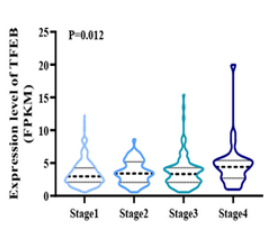

D

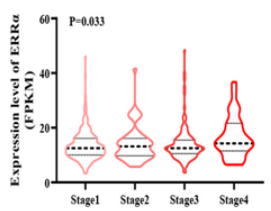

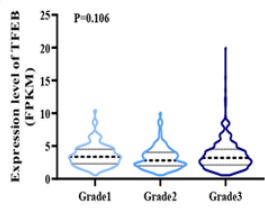

E

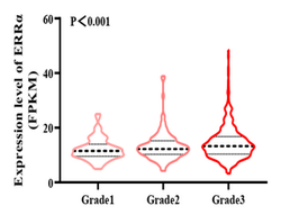

c

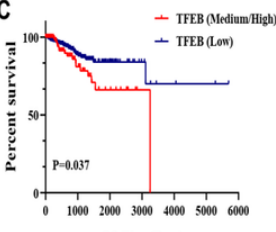

0S-Time(Days)

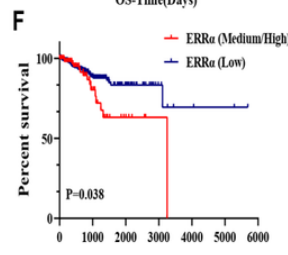

0s-Time(Days)

0
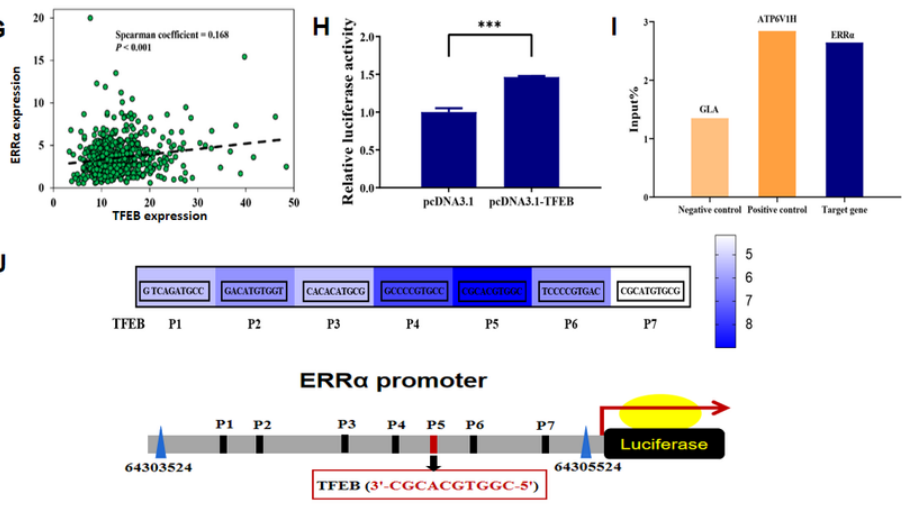

K
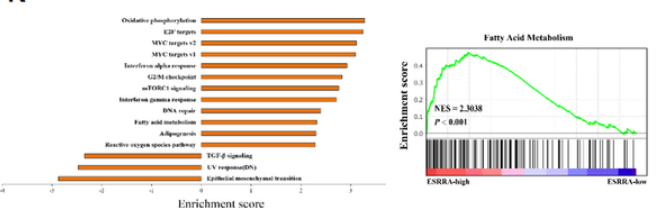

||||||||||||||||||||||||||||||

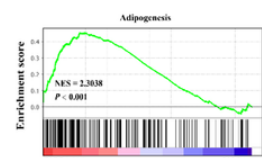

Figure 1

Bioinformatics analysis revealed that TFEB promotes ERRa transcription to participate in EC progression TCGA database [Sample size: Normal=23; EC=543] results are shown. (A) The expression of TFEB varies at different FIGO stages (B) and at different pathological grades. (C) The association of TFEB with OS in the patient/specimen quartiles is shown (Low: 1st quartile distribution; Median: 2nd-3rd quartile distribution; High: 4th quartile distribution). (D) The expression of ERRa varies at different FIGO stages (E) and at different pathological grades. (F) The association of ERRa with OS is shown. (G) The correlation between the expression levels of TFEB and ERRa in EC tissue. $(\mathrm{H})$ ChIP analysis of the ERRa promoter occupancy in KLE cells is performed as described in the Materials and Methods section. TFEB is immunoprecipitated using an anti-FLAG antibody, and DNA enrichment is performed using qPCR. The ATP6V1H promoter is used as a positive control and the GLA promoter is used as a negative control. (I) KLE cells are co-transfected with Flag-TFEB, ERRa promoter labeled with luciferase reporter, and Renilla luciferase control. Forty-eight hours after transfection, the cells are analysed, and the relative luciferase activity is measured and normalized to the Renilla luciferase control. $(J)$ The putative ERRa-binding sites (ERREs), as predicted by the online program jaspar (https://jaspar.genereg.net/analysis), are located in the TFEB (P1-P7) gene promoter regulatory regions. (K) KEGG pathway analysis (Ordinate: the KEGG signal path; abscissa: enrichment score). Results of GSEA in fatty acid metabolism and adipogenesis pathways. Statistical tests: ANOVA (A-B, D-E), KaplanMeier estimator (C,F), and Pearson correlation analysis (G). P®0.05 suggests significantly different. 
A

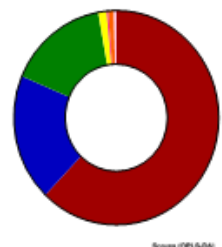

C

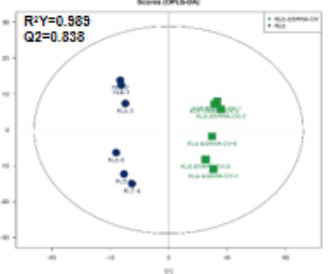

- Glycerophospholipids

- Sphingolipids

- Glycerolipids

- Fatty Acyls

Saccharolipids

B

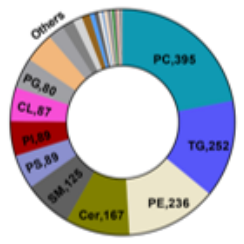

D
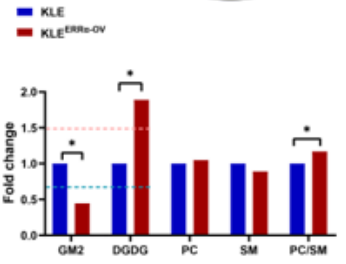

E $=$ KLE ERRo-ON $^{\text {ELe }}$
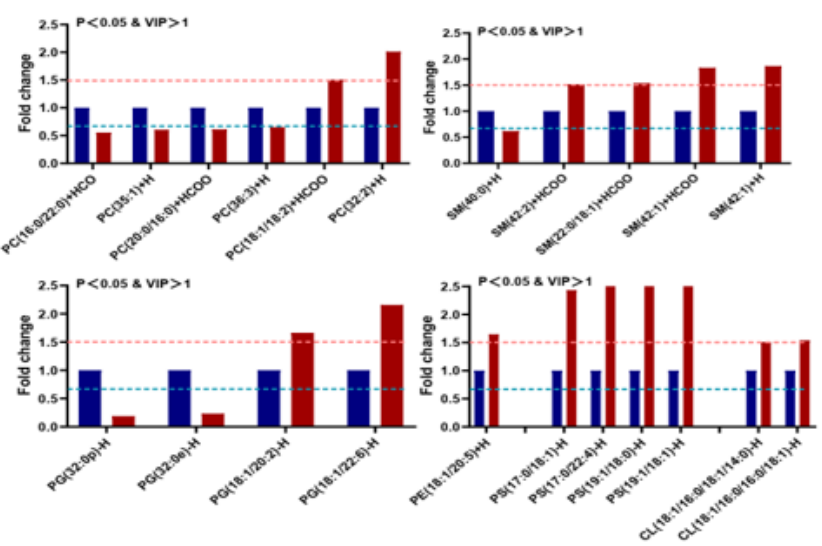

Figure 2

ERRa elevated unsaturated fatty acid (UFA)-containing GPs in EC (A) Seven subgroups of lipids in KLE cells are detected using lipidomics. (B) 36 classes of lipids were tested in KLE cells. (C) Systematic lipidomic changes between KLE and KLEERRa-OV assessed by orthogonal partial least squares-discriminant analysis (OPLS-DA). (D-E) Lipid species associated with ERRa $\left(\mathrm{p}<0.05\right.$ and VIP®1 indicate significant). ${ }^{*}, \mathrm{P}<0.05$. Statistical tests: Student' $\mathrm{s}$ t-test. 
A

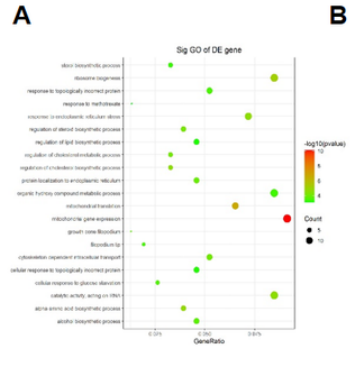

D $-\log _{10}$ (adj pvalue)
0
1

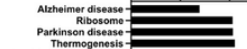

B
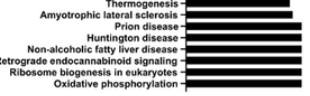

KEGG
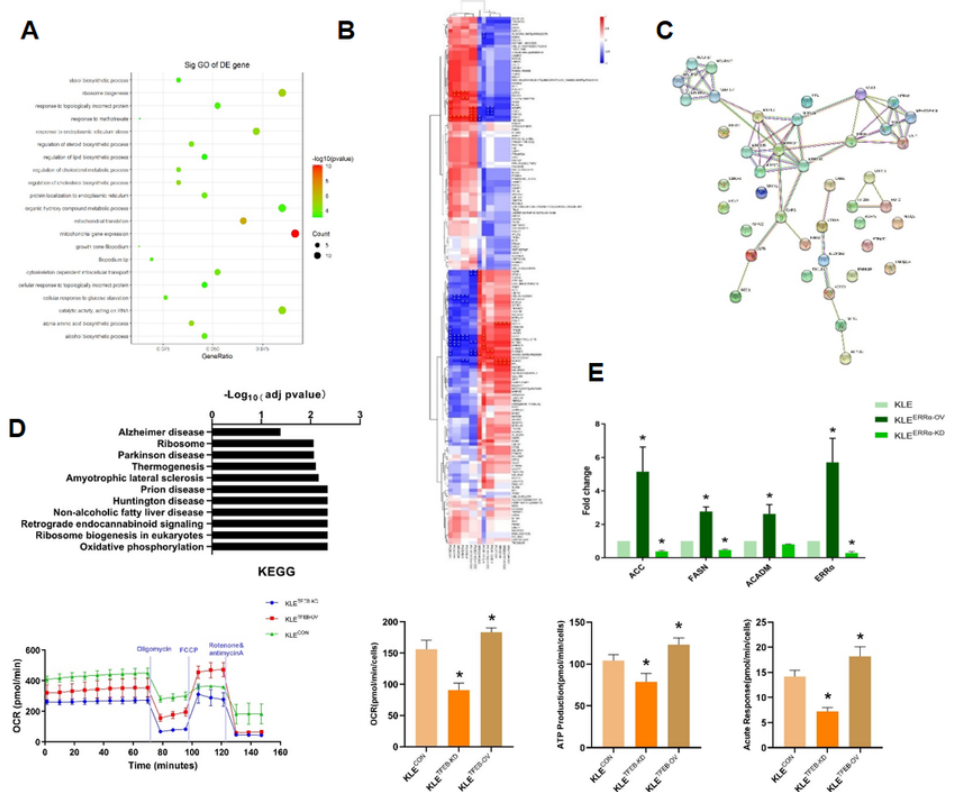

E

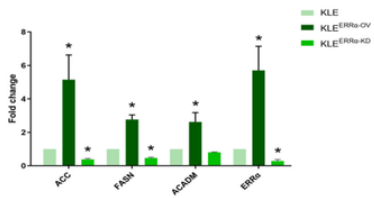

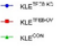
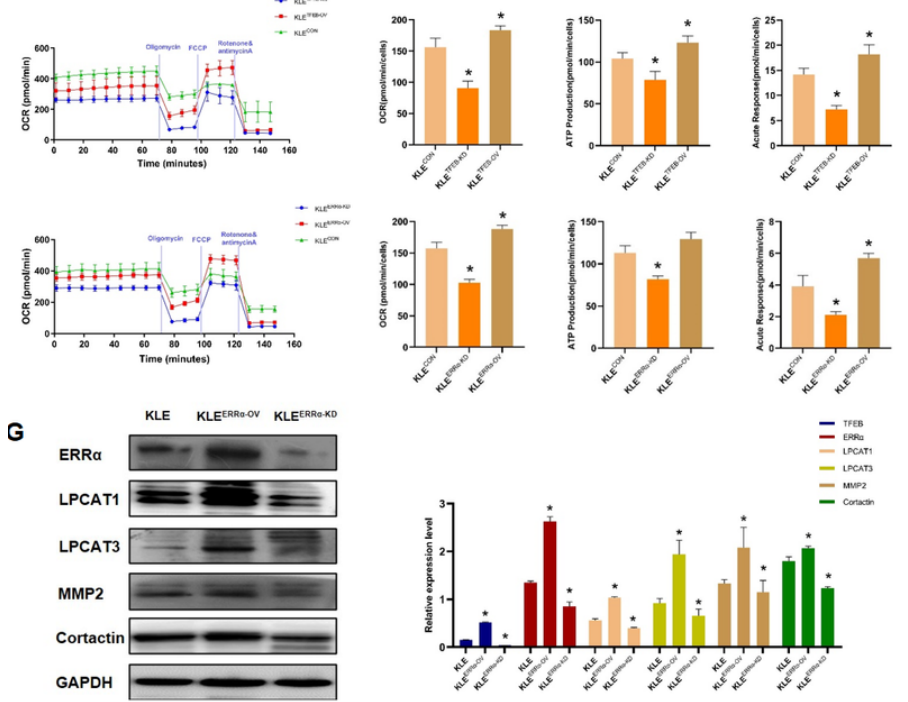

Figure 3

Proteins/lipids related to ERRa were enriched mitochondrial function of EC (A) Enrichment analysis for canonical pathways (CP) and biofunctions (BF) was performed on Proteins related to ERRa. (B) 173 Proteins related to ERRa with unique peptides $\geq 2, F C>1.3$ and $p<1.0$ were gained in KLE cells treatment with XCT790 using proteomics. (blue, down-regulated; red, up-regulated) (C) PPI identifies proteins related to ERRa with scores $>500$. (D) KEGG analysis (Ordinate: the KEGG signal path; abscissa: enrichment score) of the oxidative phosphorylation (OXPHOS) pathway. (E) The relationship between ERRa and ACC, FASN, ACADM is determined using qPCR. (F) OCR, the activities of $\beta$-oxidation, in KLE cells treated with TFEB-KD, TFEB-OV lentivirus and ERRa-KD, ERRa-OV lentivirus. (G) The effect of ERRa regulation on TFEB, LPCAT1, LPCAT3, MMP2, and Cortactin expression in EC cells is analyzed using Western blots. *, $\mathrm{P}<$ 0.05. Statistical tests: Student' s t-test or ANOVA. 
A

oh

$24 \mathrm{~h}$
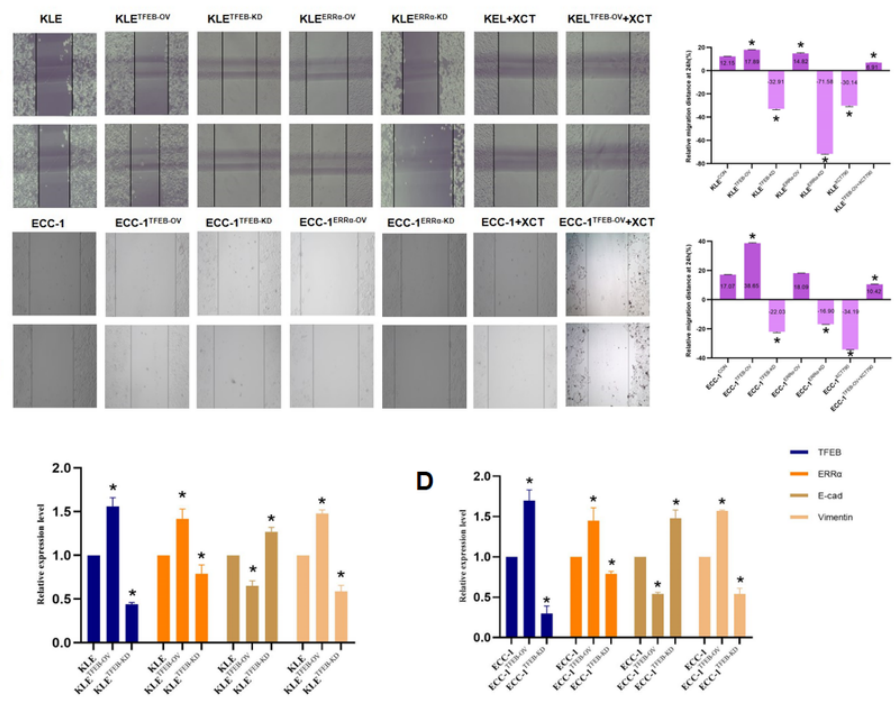

D
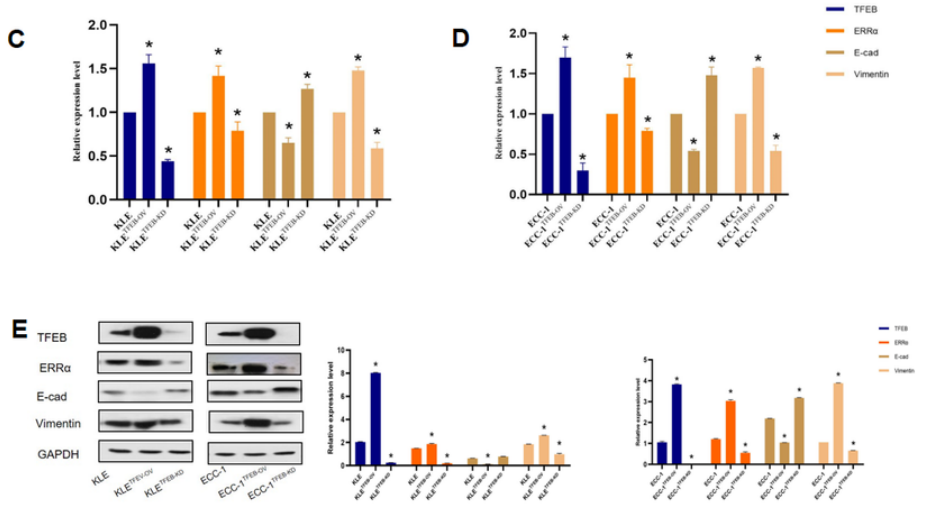

$\mathbf{F}$

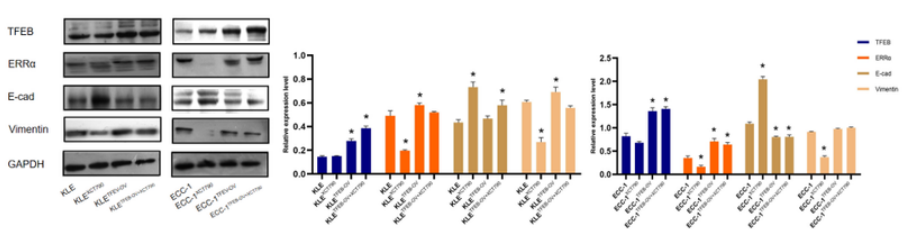

Figure 4

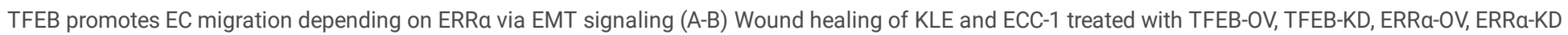

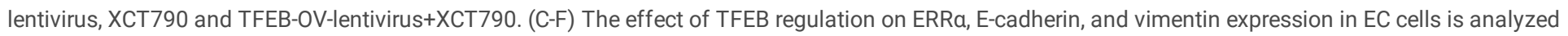
using qPCR and Western blots. *, $\mathrm{P}<0.05$. Statistical tests: Student' s t-test. 


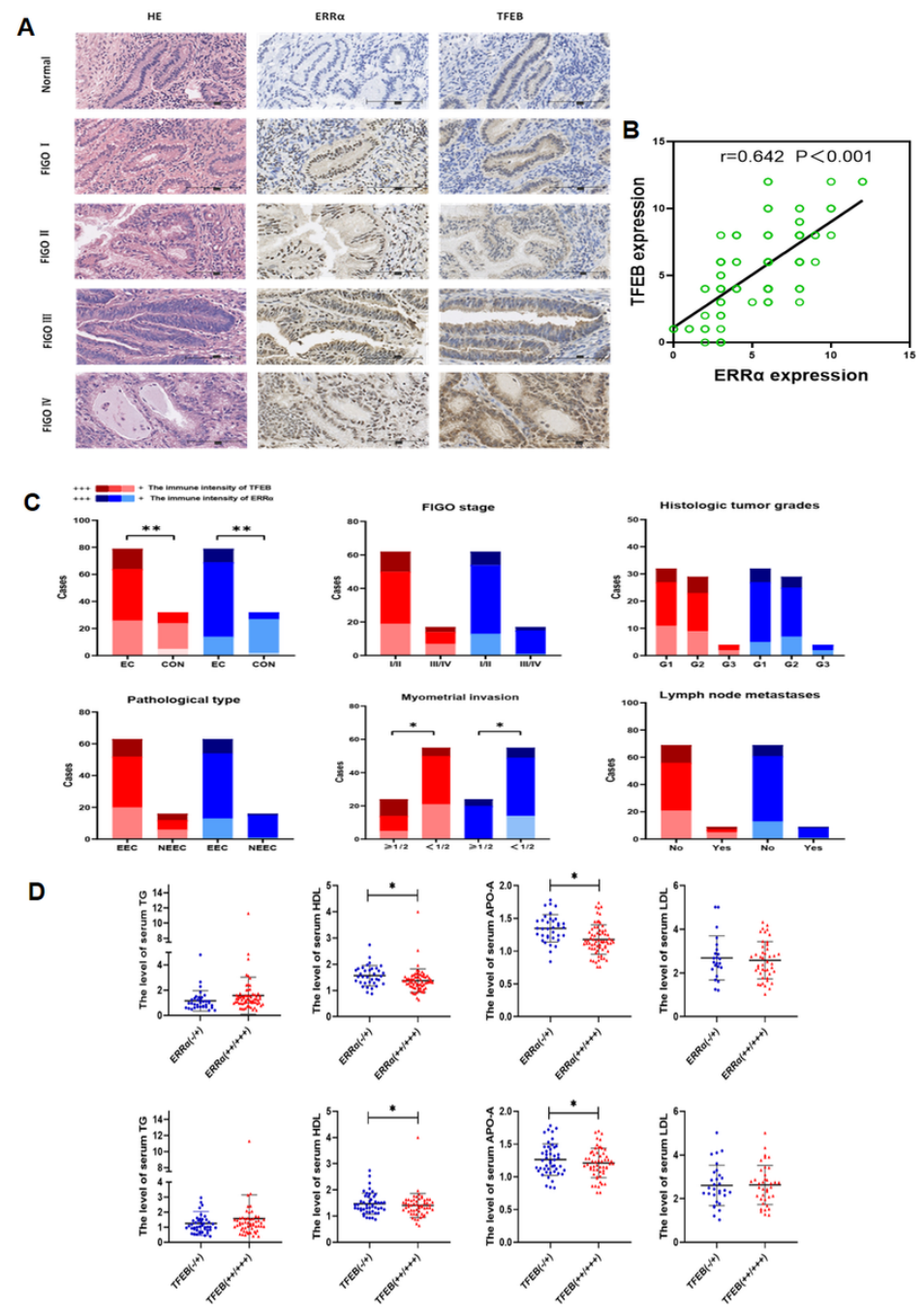

Figure 5

High expression of TFEB-ERRa is associated with dyslipidemia and metastasis in EC patients The immunohistochemical expression of several proteins and their correlations are shown. (A) The immunohistochemical expression of TFEB and ERRa in the normal endometrium ( $n=32$ ) and EC at different FIGO stages $(n=79)$ (magnification: $\times 400)$. (B) The correlation between TFEB and ERRa expression in tissues is shown. (C) The immunohistochemical scores of TFEB and ERRa in groups with different clinicopathologic features, including normal endometrium, EC, FIGO stage, histologic grade, pathological type, myometrial invasion, and lymph node metastases groups are shown. (D) The differences level of serum TG, HDL, APOA and LDL in groups with different expression levels of TFEB (TFEB-/++ vs TFEB++/+++) and ERRa (ERRa-/++ vs ERRa++/+++). *, P < 0.05. Statistical tests: Student's t-test (D), pearson's rank correlation analysis (C). 
A

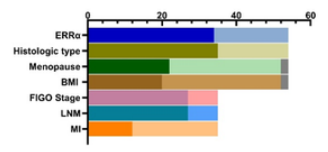

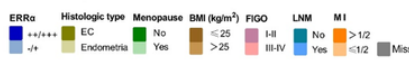

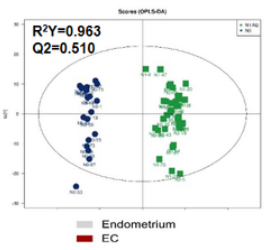

B

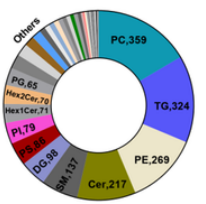

D

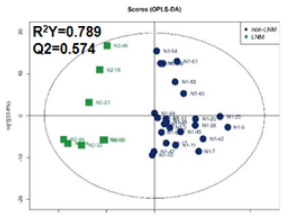

E

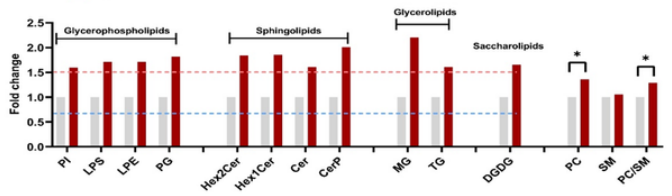

F
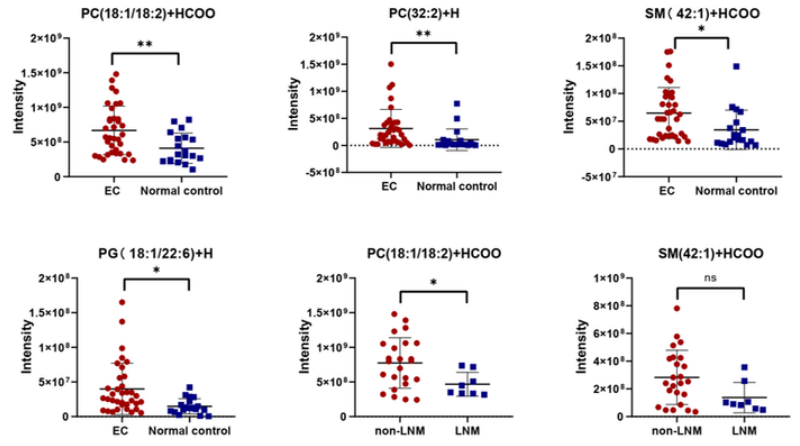

G

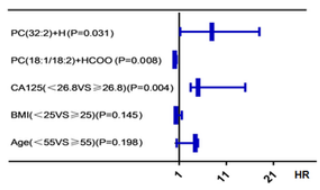

H

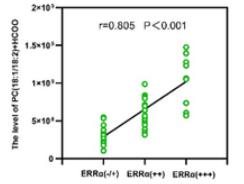

\section{Figure 6}

Accumulation of UFA-containing GPs induced by ERRa is required for EC progression (A) The baseline characteristic including BMI, menopause, FIGO stage, histologic type, MI, LNM and ERRa expression of patients ( $E C=35$ vs controls=19) tissues for lipidomic. (B) Different classes of lipids were tested in $35 \mathrm{EC}$ tissues and 19 control tissues. (C) Systematic lipidomic changes between EC and control tissues assessed by OPLS-DA. (D) Systematic lipidomic changes between LNM and non-LNM tissues assessed by OPLS-DA. (E) Lipid species in EC and normal control tissues. (F) The expression of UFA-containing GPSs, such as PC (18:1/18:2) +HCOO, PC (32:2) +H, PG (18:1/22:6) +H and SM (42:1) +HCOO were different in EC and controls. PC (18:1/18:2) +HCOO was associated with LNM. (G) The univariate binary logistic regression analyses of Age, BMI, CA125, PC (18:1/18:2) +HCOO and PC (32:2) +H. (H) The correlation between PC (18:1/18:2) +HCOO and ERRa expression is shown. *, P < 0.05. Statistical tests: Student's t-test(E-F), logistic regression(G), pearson's rank correlation analysis $(\mathrm{H})$.

\section{Supplementary Files}

This is a list of supplementary files associated with this preprint. Click to download.

- SupplementFigure.pptx

- SupplementTable1.xls 